\title{
A PANORAMIC MID-INFRARED SURVEY OF TWO DISTANT CLUSTERS
}

\author{
J. E. Geach, ${ }^{1}$ Ian Smail $^{2}{ }^{2}$ R. S. Ellis, ${ }^{3}$ S. M. Moran, ${ }^{3}$ G. P. Smith, ${ }^{3,4}$ T. Treu, ${ }^{5}$ J.-P. Kneib, ${ }^{6}$ A. C. Edge, ${ }^{2}$ and T. Kodama ${ }^{7}$ \\ Received 2006 January 24; accepted 2006 June 5
}

\begin{abstract}
We present panoramic Spitzer MIPS $24 \mu \mathrm{m}$ observations, covering $\sim 9 \times 9 \mathrm{Mpc}^{2}\left(25^{\prime} \times 25^{\prime}\right)$ fields around two massive clusters, $\mathrm{Cl} 0024+16$ and MS 0451-03, at $z=0.39$ and $z=0.55$, respectively, reaching a $5 \sigma$ flux limit of $\sim 200 \mu \mathrm{Jy}$. Our observations cover a very wide range of environments within these clusters, from high-density regions around the cores out to the turnaround radius. Cross-correlating the mid-infrared catalogs with deep optical and nearinfrared imaging of these fields, we investigate the optical/near-infrared colors of the mid-infrared sources. We find excesses of mid-infrared sources with the optical/near-infrared colors expected of cluster members in the two clusters and test this selection using spectroscopically confirmed $24 \mu \mathrm{m}$ members. The much more significant excess is associated with $\mathrm{Cl} 0024+16$, whereas MS 0451-03 has comparatively few mid-infrared sources. The mid-infrared galaxy population in $\mathrm{Cl} 0024+16$ appears to be associated with dusty star-forming galaxies (typically redder than the general cluster population by up to $A_{V} \sim 1-2 \mathrm{mag}$ ) rather than emission from dusty tori around active galactic nuclei in earlytype hosts. We compare the star formation rates derived from the total infrared $(8-1000 \mu \mathrm{m})$ luminosities for the midinfrared sources in $\mathrm{Cl} 0024+16$ with those estimated from a published $\mathrm{H} \alpha$ survey, finding rates $\gtrsim 5$ times those found from $\mathrm{H} \alpha$, indicating significant obscured activity in the cluster population. Compared to previous mid-infrared surveys of clusters from $z \sim 0-0.5$, we find evidence for strong evolution of the level of dust-obscured star formation in dense environments to $z=0.5$, analogous to the rise in the fraction of optically selected star-forming galaxies seen in clusters and the field out to similar redshifts. However, there are clearly significant cluster-to-cluster variations in the populations of mid-infrared sources, probably reflecting differences in the intracluster media and recent dynamical evolution of these systems.
\end{abstract}

Subject headings: galaxies: clusters: general — galaxies: clusters: individual (Cl 0024+16, MS 0451-03) — galaxies: starburst — infrared: galaxies

Online material: color figures

\section{INTRODUCTION}

The galaxy populations within the virialized regions of rich clusters at $z \sim 0$ are characterized by passive elliptical and lenticular (S0) galaxies (Oemler 1974; Dressler 1980). In contrast, 5 Gyr ago at $z \sim 0.5$, the galaxy populations in the most massive clusters had larger fractions of star-forming late-type spiral galaxies and a corresponding deficit of luminous S0 galaxies (e.g., Dressler et al. 1997; Smail et al. 1997; Couch et al. 1998; Fasano et al. 2000; Treu et al. 2003). Taken together, these two observations imply that a process (or processes) is transforming many of the star-forming, late-type spiral galaxies in these regions into the passive early-type population (specifically S0s) found in local clusters (Poggianti et al. 1999; S. M. Moran et al. 2006 , in preparation).

When considering potential pathways to produce this evolutionary change, we need to bear in mind that the typical lu-

\footnotetext{
1 Department of Physics, Durham University, South Road, Durham DH1 3LE, UK; j.e.geach@durham.ac.uk.

${ }^{2}$ Institute for Computational Cosmology, Durham University, South Road, Durham DH1 3LE, UK.

3 Department of Astronomy, California Institute of Technology, MC 105-24, Pasadena, CA 91125.

4 School of Physics and Astronomy, University of Birmingham, Edgbaston, Birmingham B15 2TT, UK.

5 Physics Department, University of California, Broida Hall, Mail Code 9530, Santa Barbara, CA 93106-9530.

6 OAMP, Laboratoire d'Astrophysique de Marseille, UMR 6110 Traverse du Siphon, 13012 Marseille, France.

7 Optical and Infrared Astronomy Division, National Astronomical Observatory, Mitaka, Tokyo 181-8588, Japan.
}

minosities of the star-forming spiral galaxies appear to be too low for them to transform into the typical $\mathrm{S} 0$ galaxies found in local clusters without the addition of significant numbers of new stars (Poggianti et al. 1999; Kodama \& Smail 2001). This problem is exacerbated when we include the fading that is likely to take place after the cessation of star formation in these galaxies. This then leads us to concentrate on mechanisms that are capable of increasing the luminosity of the galaxies: mergers and starbursts. There has been a recent upsurge in interest in the potential for so-called dry mergers (mergers between dissipationless stellar systems that do not result in additional star formation) to influence the evolution of early-type galaxies (van Dokkum \& Ellis 2003; Bell et al. 2003; van Dokkum 2005). However, the dynamically hot environments in rich clusters, which are the subject of our study, are deleterious to the formation and survival of cold, bound pairs of early-type galaxies (unless these systems arrive in the cluster as existing bound entities). It is not clear, therefore, that dry mergers can provide an effective route to substantially increasing the number of luminous, early-type S0 galaxies within clusters.

Unfortunately, the alternative mechanism for enhancing the luminosity of the bulge component (a starburst) also has strong observational evidence stacked against it. Surveys of star-forming galaxies in clusters using optical or UV star formation indicators have failed to detect galaxies with strongly enhanced star formation, which would have to exist to explain the growth of the bulge components of early-type galaxies in clusters at $z \lesssim 0.5-1$ (Balogh et al. 1999; Poggianti et al. 1999; Gerken et al. 2004). However, there is a growing body of evidence that at least some of the galaxies in distant clusters may be undergoing bursts of 
star formation, albeit ones that are heavily shrouded in dust. Smail et al. (1999) used a deep Very Large Array (VLA) 1.4 GHz radio map to study a small sample of active galaxies within the core of the cluster $\mathrm{Cl} 0939+4713(z=0.41)$. Combining the radio data with near-infrared and optical morphological information from the Hubble Space Telescope (HST) and ground-based spectroscopy, they found that over half the radio-emitting population in the core are dusty late-type galaxies, presumably undergoing vigorous star formation. However, the spectral classification of these spiral galaxies placed them in the poststarburst class, and indeed all the poststarburst galaxies in this small region are radio emitters. Dust has also been used to explain the unusual spectral properties of another class of galaxies found in distant clusters and the field, e(a) galaxies, which show enhanced Balmer absorption compared to normal star-forming galaxies (Poggianti et al. 1999). Poggianti \& Wu (2000) and Poggianti et al. (2001) discuss models for these galaxies, invoking age-dependent dust obscuration of the younger stellar populations, enabling significant activity to be hidden from view in these systems. If the passive lenticular galaxies found in local clusters but absent from the equivalent rich environments at higher redshift are the result of infalling latetype galaxies undergoing dusty starburst in high- $z$ clusters, then a possible signature would be evolution in the total level of obscured star formation in clusters out to $z \sim 1$.

In principle, mid- and especially far-infrared/submillimeter observations give us a direct probe of the level of obscured activity in distant clusters. In particular, mid-infrared observations with the Infrared Space Observatory (ISO) and, more recently, the Spitzer Space Telescope provide sensitive imaging capabilities that can trace dusty star formation in clusters out to $z \sim 1$ and beyond. Metcalfe et al. (2005) summarize the results from ISO surveys of distant clusters, which have yielded a total of just $\sim 40$ cluster galaxies detected at $15 \mu \mathrm{m}$ across seven clusters between $z=0.18$ and 0.56 (Duc et al. 2000, 2004; Fadda et al. 2000; Metcalfe et al. 2003; Coia et al. 2005; Biviano et al. 2004). All these studies suggest that there is an increased level of midinfrared activity in distant clusters, at levels above that suggested by UV/optical tracers of star formation. Submillimeter observations of more distant clusters have also hinted at possible enhanced activity in these environments (Best 2000; Webb et al. 2005). However, the inhomogeneous mix of coverage and depth in the samples coupled with the modest numbers of sources detected in any individual cluster mean that it has proved difficult to use these data to provide quantitative constraints on the origin and evolution of dust-obscured activity in distant clusters.

Spitzer's sensitive mid-infrared imaging capabilities provide a unique opportunity to undertake complete and representative surveys of the obscured, active populations in distant clusters. To search for a population of mid-infrared sources in rich cluster environments, we have therefore used the Multiband Imaging Photometer of Spitzer (MIPS) to detect $24 \mu \mathrm{m}$ emission from galaxies in two clusters at $z \sim 0.5$, covering a very wide range in environment from $\sim 1 \mathrm{Mpc}$ out to the turnaround radius ( $\sim 5 \mathrm{Mpc})$, where the clusters merge into the surrounding field. These observations provide measures of the level of obscured star formation in these clusters, and so allow us to build up a reliable picture of the evolution of dust-obscured activity in clusters over the past 5 Gyr.

This paper presents a statistical analysis of the $24 \mu \mathrm{m}$ populations in the two $z \sim 0.5$ clusters. A subsequent paper (J. E. Geach et al. 2006, in preparation) will discuss the properties of these sources in more detail, using the available spectroscopic and morphological surveys of the clusters (Moran et al. 2006). This paper is organized as follows: we describe our observa- tions and their reduction in $\S 2$, analyze them in $\S 3$, and discuss our results and present our conclusions in $\S \S 4$ and 5 , respectively. Throughout, we adopt a geometry with $\Omega_{m}=0.3, \Omega_{\Lambda}=0.7$, and $H_{0}=70 \mathrm{~km} \mathrm{~s}^{-1} \mathrm{Mpc}^{-1}$.

\section{OBSERVATIONS AND REDUCTION}

The two clusters chosen for this study are unique in having panoramic HST imaging covering $\sim 25^{\prime}$ diameter fields, extending from the cores out to the turnaround radii of the clusters (Treu et al. 2003; Kneib et al. 2003; Moran et al. 2005). These data have been used for weak-lensing analysis of these clusters, yielding two-dimensional maps of the dark matter distributions on $\sim 5 \mathrm{Mpc}$ scales in the structures (Kneib et al. 2003). Panoramic studies of the galaxy populations in these clusters also benefit from extensive deep, ground-based optical and near-infrared imaging and spectroscopy (S. M. Moran et al. 2006, in preparation). Although both clusters are relatively rich, they differ in their X-ray luminosities: $\mathrm{Cl} 0024+16(z=0.39)$ has a relatively modest X-ray luminosity, $L_{X} \sim 3.2 \times 10^{44} \mathrm{ergs} \mathrm{s}^{-1}$ (Treu et al. 2003), while MS $0451-03(z=0.55)$ is some 8 times more luminous. This distinction may lead to differences in the effectiveness of the various processes influencing star formation (Treu et al. 2003), as traced by the distribution of the mid-infrared population. This is useful in our subsequent detailed study for disentangling the potential mechanisms for triggering and suppressing star formation.

\subsection{Mid-Infrared Observations}

MIPS $24 \mu \mathrm{m}$ observations of the fields of $\mathrm{Cl} 0024+16(z=0.39)$ and MS 0451-03 ( $z=0.55)$ were obtained with Spitzer in fixedcluster offset mode on 2004 December 24 and 25. The observations of $\mathrm{Cl} 0024+16$ are centered on R.A. $=00^{\mathrm{h}} 26^{\mathrm{m}} 35^{\mathrm{s}} .70$, decl. $=+17^{\circ} 09^{\prime} 45^{\prime \prime}(\mathrm{J} 2000.0)$, while those for MS 0451-03 are centered on R.A. $=04^{\mathrm{h}} 54^{\mathrm{m}} 10^{\mathrm{s}} .80$, decl. $=-03^{\circ} 00^{\prime} 57^{\prime \prime}(\mathrm{J} 2000.0)$. The observations avoided the central $\sim 5^{\prime} \times 5^{\prime}$ of each cluster, which are part of Guaranteed Time Observations (Spitzer program 83). For MS 0451-03 we have acquired these data from the archive and incorporated them into this work to provide coverage of the central region of the cluster. The observations of the core of $\mathrm{Cl} 0024+16$ were not available at the time of this writing; however there are existing mid-infrared observations from ISOCAM at $15 \mu \mathrm{m}$ (Coia et al. 2005), which traces emission similar to that sampled by MIPS (namely, emission from polycyclic aromatic hydrocarbons). These allow us to place limits on the mid-infrared population in the central regions of Cl $0024+16$.

Each field was covered in a $5 \times 5$ grid (omitting the central pointing) yielding a 24 point mosaic. We adopted two $10 \mathrm{~s}$ cycles for our exposures, producing a per astronomical observation request (AOR) pixel exposure of $312.5 \mathrm{~s}$, and the total elapsed time for the AOR was $9.78 \mathrm{ks}$. The template AOR was repeated three times to provide redundancy and for the identification of cosmic rays and asteroids (the latter can be common in $24 \mu \mathrm{m}$ MIPS data). The total exposure time per pixel is $938 \mathrm{~s}$, which should achieve our goal of a $5 \sigma$ detection limit of $200 \mu \mathrm{Jy}$ at $24 \mu \mathrm{m}$.

The basic-calibrated $24 \mu \mathrm{m}$ data provided by the Spitzer Science Center were first corrected for gradients, bright latents, and the "jailbar" effect using calibration frames generated from the data. These were then mosaicked, using the MOPEX package. ${ }^{8}$ Due to the "first-frame effect" (frames with a shorter exposure and a depression in response of up to $15 \%$ ), the initial frame in

\footnotetext{
${ }^{8}$ MOPEX is maintained by the Spitzer Science Center at http://ssc.spitzer caltech.edu.
} 
each pointing was discarded, which improved flatness in the final mosaic. We implemented the area-overlap interpolation method in the mosaicking procedure (Makovoz \& Khan 2005). The mosaics cover total areas of $\sim 0.21 \mathrm{deg}^{2}$ in both clusters.

Source extraction was performed using SExtractor version 3.1 (Bertin \& Arnouts 1996), with the criteria that a source consist of at least three contiguous pixels (each pixel is 2 .'5 square) at $\geq 2 \sigma$ above the background. We measure $16^{\prime \prime}$ diameter aperture fluxes, corresponding to $\sim 3$ times the FWHM of the point-spread function (PSF; FWHM of $\sim 5^{\prime \prime}$ at $24 \mu \mathrm{m}$ ). Using a curve-of-growth analysis on bright isolated point sources, and our completeness simulations, we find that $16^{\prime \prime}$ apertures recover $\sim 75 \%$ of the total flux, and we therefore correct the resulting fluxes by a factor of 1.33 to yield the total fluxes. We visually inspect the extracted objects overplotted on the images and remove sources that appear to be false, e.g., in the slightly noisier regions on the edge of the image.

The flux detection limits and completeness of each mosaic were determined by inserting 10 sets of 10 simulated sources into the mosaics for a range of flux and determining their detection rate and recovered fluxes, using identical extraction parameters. The sources used in the simulations were high signal-to-noise ratio $(\mathrm{S} / \mathrm{N})$ composites formed by stacking a large number of real, isolated faint sources. From these simulations, we estimate that the $80 \%$ completeness limits of our observations are $180 \mu \mathrm{Jy}$ in $\mathrm{Cl} 0024+16$ and $200 \mu \mathrm{Jy}$ in MS 0451-03.

The archival mid-infrared data for the central region of MS 0451-03 (program 83) were obtained from the Spitzer archive. The observations were taken on 2004 September 23, centering on R.A. $=04^{\mathrm{h}} 54^{\mathrm{m}} 10^{\mathrm{s}} 8$, decl. $=-03^{\circ} 00^{\prime} 57^{\prime \prime}(\mathrm{J} 2000.0)$. We use the post-basic-calibrated data and subject it to the same extraction criteria as described in $\S 2.1$. These data are slightly shallower than our mosaic, and we take this into account in our subsequent analysis.

\subsection{Archival Optical and Near-Infrared Imaging}

We use existing deep optical and near-infrared imaging to obtain colors for the $24 \mu \mathrm{m}$ sources in these regions. For both clusters, we use Subaru SuprimeCam $B$ - and $R$-band observations taken for the PISCES survey (Kodama et al. 2005). The $\mathrm{Cl}$ 0024+16 field was observed on 2002 September 6, under good conditions. The seeing was $\sim 0.7-1^{\prime \prime}$ for the $R$-band and $\sim 1^{\prime \prime}-1$." 3 for the $B$-band, with exposure times of 5280 and $3600 \mathrm{~s}$, respectively, reaching a depth of $R \sim 27$ (we use Vega-based magnitudes throughout). The observations and data-reduction technique are described in Kodama et al. (2004). MS 0451-03 was observed on 2001 January 21 and 22, again in good conditions, with 1".0 seeing in the $B$ band and 0.8 in the $R$ band. Total exposure times were $7200 \mathrm{~s}$ in $B$ and $4800 \mathrm{~s}$ in $R$, again yielding photometry for objects as faint as $R \sim 27$.

Panoramic near-infrared $K$-band imaging of both clusters is also available with the Wide-Field Infrared Camera (WIRC; Wilson et al. 2003) on the Palomar Hale $5.1 \mathrm{~m}$ telescope. These data comprise a $3 \times 3$ mosaic of WIRC pointings, providing a contiguous observed area of $26^{\prime} \times 26^{\prime}$ centered on each cluster. Full details of the observations and data reduction are published elsewhere ( $\mathrm{Cl} 0024+16$ : Smith et al. 2005; MS 0451-03: G. Smith et al. 2006, in preparation). Point sources in the final reduced mosaics have a full width at half-maximum of 0.9 and 1.0 in $\mathrm{Cl} 0024+16$ and MS 0451-03, respectively. The data reach a $5 \sigma$ point-source detection threshold of $K=19.5$ and 20.0 in $\mathrm{Cl} 0024+16$ and MS 0451-03, respectively. The nominal $24 \mu \mathrm{m}$ coverage of the two clusters is $\sim 0.21 \mathrm{deg}^{2}$. In the case of MS $0451-03$, the archival GTO data contribute an extra $\sim 29 \operatorname{arcmin}^{2}$.
When the optical and near-infrared coverage is taken into account, the total coincident coverage of $24 \mu \mathrm{m}, B, R$, and $K$ bands is 0.165 and $0.184 \mathrm{deg}^{2}$ in $\mathrm{Cl} 0024+16$ and MS 0451-03, respectively.

We detect objects and extract photometry from the optical frames by running SExtractor in two-frame mode, such that detections were made in the $R$-band image and measurements were made at identical pixel locations in $R$ and $B$ (the frames were previously aligned to good accuracy). We catalog all sources with three contiguous pixels (the pixel scale is 0.204 ), at least $2 \sigma$ above the background, and lay down $2^{\prime \prime}$ diameter apertures to measure colors in the $B R K$-band frames.

\section{ANALYSIS AND RESULTS}

The MIPS observations of our two clusters yield 986 sources in the $\mathrm{Cl} 0024+16$ field and 1071 in MS 0451-03, both with $24 \mu \mathrm{m}$ flux densities above $S_{24} \mu \mathrm{m}>200 \mu \mathrm{Jy}$ (unless otherwise stated, quoted fluxes are always the corrected $16^{\prime \prime}$ aperture values). In the case of MS 0451-03, the count includes sources in the archival region in the core. We assess the false detection rate by running our algorithm on the inverse of the data and detect $\sim 20$ sources in each frame, all with fluxes in the range 200-330 $\mu \mathrm{Jy}$, representing $\sim 2 \%$ of the detected sample. Our analysis requires matched optical $R<24$ identifications, so we expect the false rate to be much less than $1 \%$. As we demonstrate below, the achieved source surface density is below 1 object per 40 beams, the classical definition of a confused map (Hogg 2001), so our maps are not expected to be confused at this depth.

We next use the deep optical and near-infrared imaging of these fields to investigate the photometric properties of mid-infrared sources. The influence of false detections described above is minimized in the main part of our analysis, since we require the midinfrared sources to have optical and near-infrared counterparts.

\subsection{Optical Properties}

Positional matching of multiwavelength data sets has been a long-standing problem in astronomy, and can be particularly troublesome when there is a large disparity in the resolution and sampling in two data sets. We find that a simple nearestneighbor match is not adequate to pair mid-infrared sources with optical counterparts; the method can fail to match complicated interacting systems, for example. Instead, we apply the technique of de Ruiter et al. (1977), who use a Bayesian estimator for the probability, $p(\mathrm{id} \mid r$ ), that a nearby source is a true match and not a chance unrelated object. In the Appendix we briefly outline the key elements of the method but refer the reader to de Ruiter et al. (1977) for a thorough derivation. The result of our matching analysis is a list of probable optical counterparts for the $24 \mu \mathrm{m}$ sources in our catalogs. We identify 611 and 650 counterparts to $24 \mu \mathrm{m}$ sources that are brighter than $R=24$ and $200 \mu \mathrm{Jy}$ in $\mathrm{Cl} 0024+16$ and MS $0451-03$, respectively. In MS 0451-03 this includes sources in the archival region.

In Figure 1 we plot the $(B-R)$ versus $R$ color-magnitude diagram for each cluster, comparing the distribution of colors for the mid-infrared sources and their apparent magnitudes with the optical population in these regions. The first thing to note is the broad similarity in the distributions on the color-magnitude plane of the $24 \mu \mathrm{m}$ sources in the two fields. Looking at the color distributions in more detail, the median $(B-R)$ color of midinfrared sources at $R<22$ is 1.75 and 1.80 for $\mathrm{Cl} 0024+16$ and MS 0451-03, respectively. The $24 \mu \mathrm{m}$ counterparts exhibit a broad peak in $(B-R)$ color, which lies between the peaks of red and blue galaxies (Fig. 1). This association of $24 \mu \mathrm{m}$ sources with galaxies having transition colors is intriguing. It may simply reflect the fact that these galaxies are dustier examples of the 

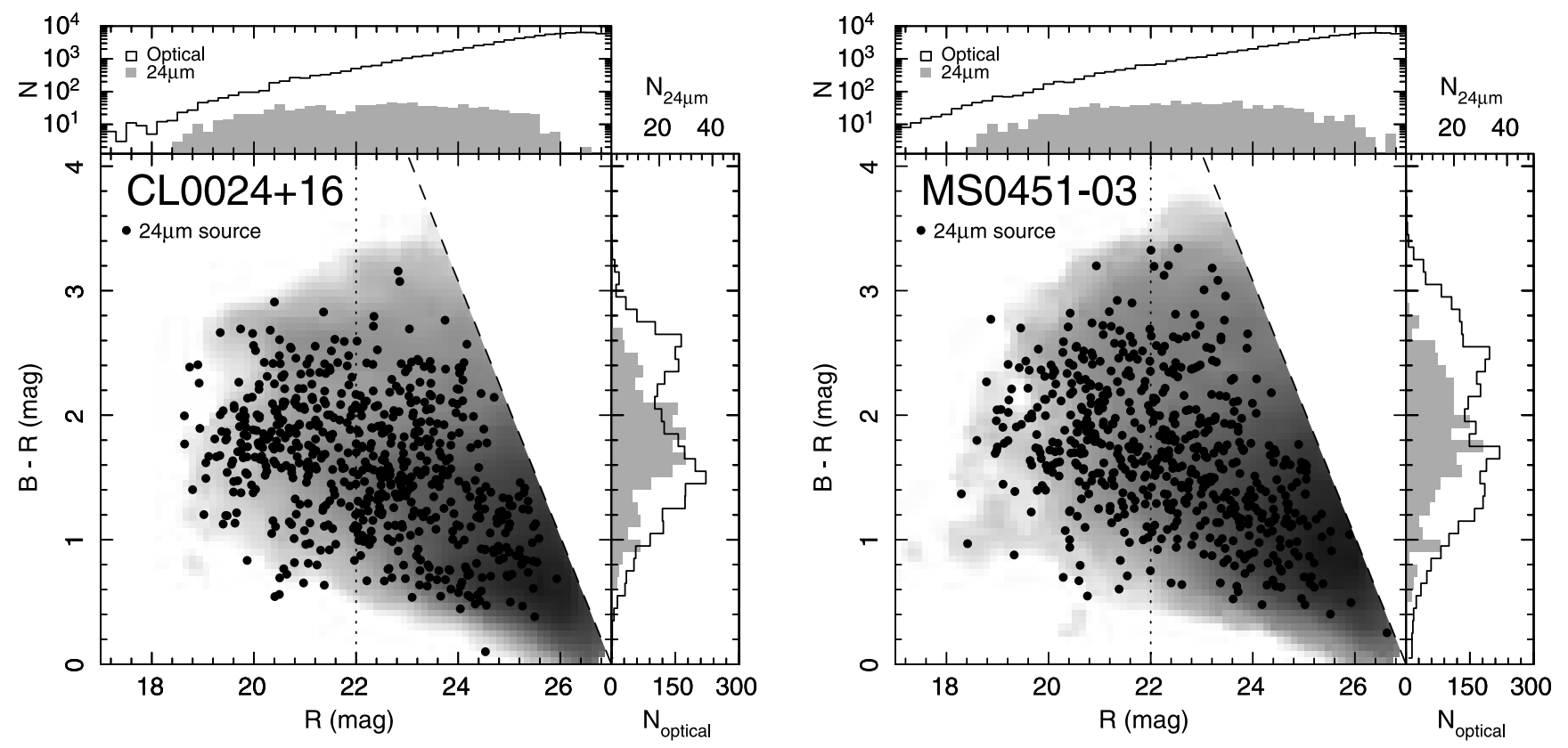

FIG. 1.- $(B-R)-R$ color-magnitude diagram for $\mathrm{Cl} 0024+16$ (left) and MS $0451-03$ (right). We identify $24 \mu \mathrm{m}$ sources brighter than $200 \mu \mathrm{Jy}$ and compare them to the distribution for the optically selected populations in these fields (for clarity, this has been represented by a smoothed density plot). Also shown are histograms showing slices through the distributions, comparing the number counts and colors for the mid-infrared sources to the optically selected population. To emphasize the location of the sequence of early-type galaxies in the clusters, the $(B-R)$ histogram is limited to $R<22$. The mid-infrared-selected population lies between the red and blue galaxy peaks in these fields, most likely because of the influence of dust on intrinsically blue, star-forming galaxies. For the mid-infrared sources in the color-magnitude diagram, we have removed those objects morphologically classified as stars. All colors are measured in $2^{\prime \prime}$ diameter apertures, and we use the BEST_MAG estimate of the total galaxy magnitude.

general blue star-forming field population, where dust reddening produces somewhat redder colors. Or it may be evidence that some of these galaxies are part of an evolutionary sequence, connecting the blue star-forming population and the passive types inhabiting the red sequence.

In Figure 2, we plot the $(R-K)$ versus $(B-R)$ colors of the mid-infrared sources in $\mathrm{Cl} 0024+16$ and MS 0451-03, compared to the optically selected populations in the two fields. We also indicate the expected colors of cluster members with a range of spectral types (King \& Ellis 1985). These model colors provide a rough guide to the relative level of current to past star formation in the galaxies, and the reader should note that the influence of dust extinction will move galaxies parallel to this sequence, further complicating any interpretation of the star formation histories of these galaxies.

Looking at the panel for $\mathrm{Cl} 0024+16$ in Figure 2, we see a clear ridge of $24 \mu \mathrm{m}$ sources, with colors comparable to those expected for cluster galaxies. To confirm this association, we indicate on the plots the locations of spectroscopically confirmed $24 \mu \mathrm{m}$ cluster members from the surveys of Czoske et al. (2001) and Moran et al. (2006), and our ongoing spectroscopic follow-up of our $24 \mu \mathrm{m}$ sample (J. E. Geach et al. 2006, in preparation). There are 45 confirmed $24 \mu \mathrm{m}$ members in $\mathrm{Cl} 0024+16$ and 7 in MS 0451-03, and the vast majority of these lie close to the predicted color-color locus for cluster members and have colors consistent with starforming galaxies. However, a few fall into the region populated by passive, early-type cluster members, as expected due to the likely presence of active galactic nuclei (AGNs) in some of these galaxies. Nevertheless, it is clear that the information from this $(B-R)-(R-K)$ color-color plot can be used to crudely isolate likely $24 \mu \mathrm{m}$ cluster members to statistically gauge the size of this population in the two clusters.

The "transitional" colors of the majority of $24 \mu \mathrm{m}$ sources hinted at in the color histograms in Figure 1, are also reflected in the distribution of points around the spectral classes in Figure 2.
The colors of the bluer, probable $24 \mu \mathrm{m}$ cluster members suggest that these are moderately star-forming galaxies (matching the continuum shapes of present-day Sbc/Scd galaxies). However, these could be more actively star-forming systems with additional reddening, $A_{V} \sim 0-1$, and there are a small number of galaxies with very red colors, which suggests very significant reddening, i.e., $A_{V} \sim 2$ mag. To select the majority of infrared cluster members, we apply a photometric cut in color-color space around the sequence of known cluster members (Fig. 2) and then account for the residual field contamination using a similar color selection applied to a blank-field $24 \mu \mathrm{m}$ sample. Based on the existing spectroscopic samples of $24 \mu \mathrm{m}$ sources in the two clusters, we estimate that our color-selection criteria identify 39 of 45 and 5 of 7 members in $\mathrm{Cl} 0024+16$ and MS 0451-03, respectively, or an average completeness of $85 \%$. We note that the fact that the reddening vector runs approximately parallel to our selection box in Figure 2 means that it should contain a significant fraction of the more reddened sources.

In addition, we investigate the nature of the star-forming cluster population, so we must be aware of contamination by infrared activity caused by AGNs in passive (and also star-forming) galaxies. Therefore, we define a subregion in the color space around the likely color of cluster E/S0s (Fig. 2) and do not include the galaxies within this region in our analysis of the obscured starforming population.

The $24 \mu \mathrm{m}$ galaxy population in the MS 0451-03 field does not exhibit such a pronounced ridge, with colors matching those expected for cluster members, as $\mathrm{Cl} 0024+16$. Nevertheless, there is a weak overdensity, which is most obvious in a red clump associated with passive, early-type cluster members. This clump is around 0.2 mag redder in $(B-R)$ and $(R-K)$ than the equivalent feature in $\mathrm{Cl} 0024+16$, as expected from the $k$-correction for passive galaxies between $z=0.39$ and 0.55. Again, the small number of spectroscopically confirmed $24 \mu \mathrm{m}$ members fall close to the locus expected for the colors of cluster members, and we 

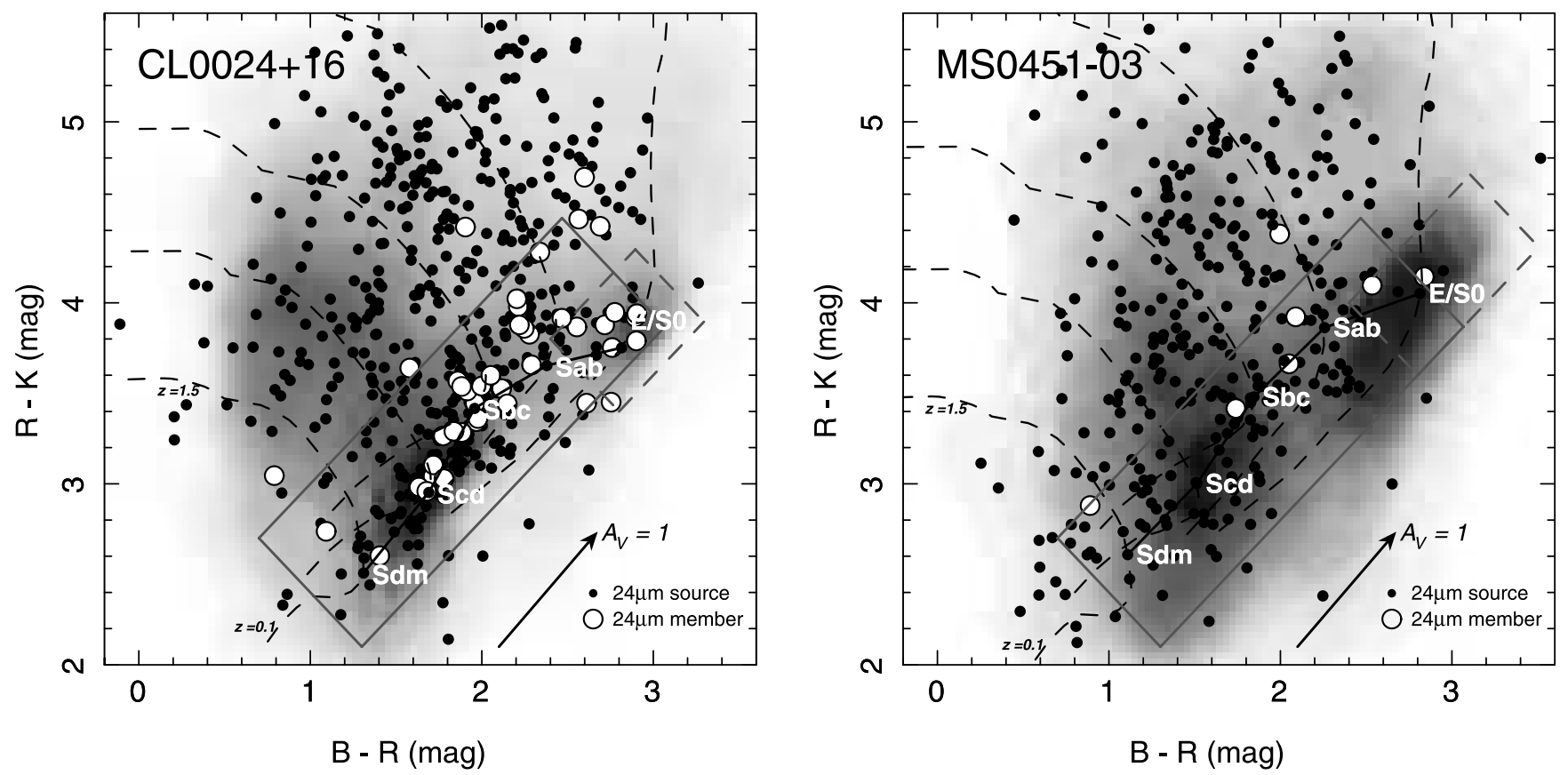

FIG. 2.- $(R-K)$ vs. $(B-R)$ colors for $24 \mu \mathrm{m}$-detected sources compared to the optical distribution in the $\mathrm{Cl} 0024+16$ (left) and MS $0451-03$ (right) fields. The distributions are limited to $R \leq 24$ and $K \leq 20$, and we represent the optical sample's number density as a slightly smoothed gray scale. The dashed tracks trace the expected colors of galaxies across $z=0-2$, with star formation histories similar to those of present-day Sdm-E galaxies, and the solid line and filled circles show the location in colorcolor space of the range of spectral types at the cluster redshift. The arrow indicates the translation in color-color space, corresponding to an increase in reddening of $A_{V}=1$. To perform a rough photometric cut to select $24 \mu \mathrm{m}$ sources at the cluster redshift, we define a region around the predicted colors of cluster members, indicated by the rectangular selection boxes in both panels. To avoid contamination by infrared emission from AGNs, we designate a subregion that should contain the passive galaxies (dashed rectangle); $24 \mu \mathrm{m}$ sources in this region are not included in our analysis of the obscured star-forming populations. We show the efficacy of this selection by plotting the colors of known spectroscopically confirmed $24 \mu \mathrm{m}$ cluster members, $85 \%$ of which fall within the selection box. [See the electronic edition of the Journal for a color version of this figure.]

therefore use this color-color selection in the subsequent sections to investigate the properties of this population.

\subsection{Counts of Mid-Infrared Sources}

To quantify the number of $24 \mu \mathrm{m}$ sources associated with cluster members, we first compare the $24 \mu \mathrm{m}$ number counts for each cluster to counts from the literature for large-area $24 \mu \mathrm{m}$ field surveys (e.g., Marleau et al. 2004; Papovich et al. 2004; Chary et al. 2004). The counts in the literature all agree well in the flux density regime covered by our data, and we use the model presented in Marleau et al. (2004) to estimate the number of field $24 \mu \mathrm{m}$ galaxies expected with $S_{24} \mu \mathrm{m}=200-1000 \mu \mathrm{Jy}$ in our fields; for $d N / d S=(416 \pm 3) S^{-2.9 \pm 0.1}$, which is valid for the range $200-900 \mu \mathrm{Jy}$, we extrapolate the bright end to $1000 \mu \mathrm{Jy}$. For this comparison, we also correct our counts for completeness in the range 200-300 $\mu \mathrm{Jy}$ (our simulations show that our observations are $\sim 100 \%$ complete at $300 \mu \mathrm{Jy}$ ).

The predicted numbers of $24 \mu \mathrm{m}$ sources to our flux limits over the $\mathrm{Cl} 0024+16$ and MS 0451-03 fields are $1280 \pm 160$ and $939 \pm 120$, respectively, down to $200 \mu \mathrm{Jy}$. These should be compared to the observed numbers of sources of 1032 and 935 , respectively. Based on this comparison we can see that the cluster populations do not represent a significant excess over the large field population, across the full survey area and down to the flux limits of our survey. This is not particularly surprising, as the survey fields are large, and our sensitivity limit is sufficient to detect sources in a large volume out to high redshifts. Instead, if we restrict the comparison to the central regions of the clusters, where the contrast of the cluster over the background might be largest, we see that, at projected radii of $\lesssim 2 \mathrm{Mpc}$, there is a slight excess of mid-infrared sources compared to the field counts in $\mathrm{Cl}$ 0024+16: $134 \pm 12$ observed sources compared to $115 \pm 14$ predicted for the field, suggesting a modest cluster population. In MS 0451-03, including the archival data for the core, we find that within $2 \mathrm{Mpc}$ there are $104 \pm 10$ sources, compared to $108 \pm 13$ predicted by the model, showing no detectable overdensity in this cluster even in the central regions.

The lack of a detectable excess in the raw counts of $24 \mu \mathrm{m}$ sources in the cluster fields does not rule out a significant cluster population, given the large uncertainties in the contribution from field sources. To provide a more sensitive test, we can exploit the optical/near-infrared photometry to remove the bulk of the background field contamination and so reduce the uncertainty in the field correction. In Figure 3 we plot the cumulative number of mid-infrared galaxies as a function of clustocentric radius for those galaxies with optical/near-infrared counterparts whose colors lie close to the ridgelines of cluster members seen in Figure 2 (restricted to those with $R<24$ ). These are corrected for residual field contamination by using the optical/near-infrared photometry of mid-infrared sources from the SWIRE ELAIS N1 survey $^{9}$ (Lonsdale et al. 2003, 2004). To achieve this, we translate our $(B-R)-(R-K)$ selection box in Figure 2 to the equivalent region in $(r-z)-(g-r)$ space for the SWIRE sample, using model spectral energy distributions (SEDs; Fig. 2) to transform between the various colors. Extrapolating the number counts of these color-selected sources to our $200 \mu \mathrm{Jy}$ limit (by fitting a power-law, $d N / d S \propto S^{-\alpha}$, to the counts) yields an average surface density of mid-infrared field sources with $R<24$ of $0.16 \pm 0.02 \mathrm{arcmin}^{-2}$, and we correct our counts using this. We also make use of the very large SWIRE survey area to estimate the fluctuations in the field correction in the area of each of our radial annuli in Figure 3, by determining the fluctuation in the

\footnotetext{
${ }^{9}$ See Surace, J. A., et al. 2004, VizieR Online Data Catalog, 2255.
} 


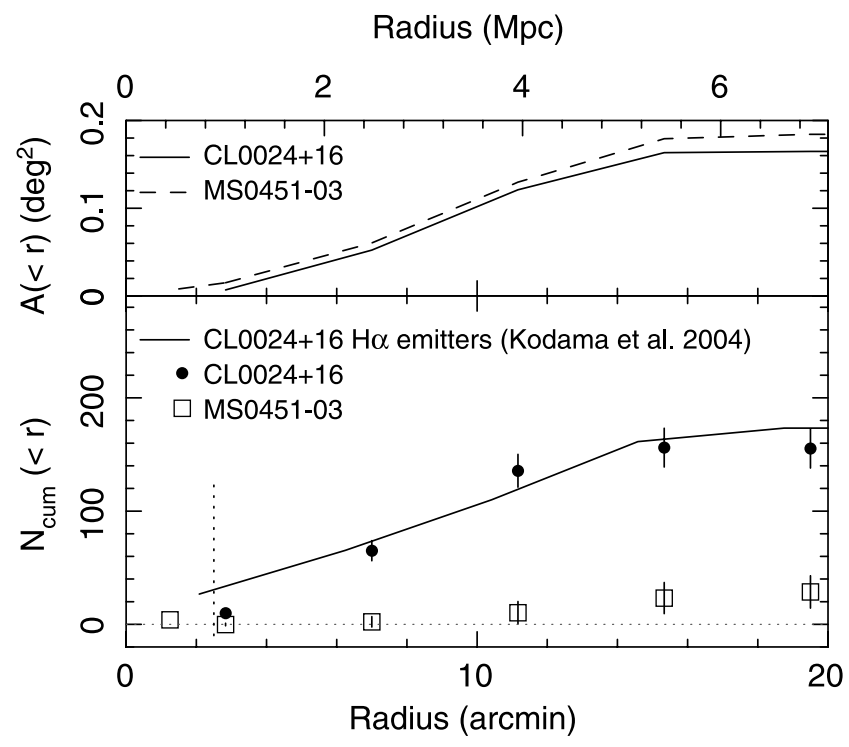

FIG. 3.-Bottom: Cumulative number of probable $24 \mu \mathrm{m}$ cluster members (brighter than $200 \mu \mathrm{Jy}$ ) as a function of radial separation from their respective cluster centers. Cluster members are probably isolated based on their $(R-K)$ $(B-R)$ colors (see Fig. 2). We correct the distributions for residual field contamination using a similar color cut on the SWIRE ELAIS N1 survey (see footnote [9]; Lonsdale et al. 2003, 2004), giving an estimated field correction of $\sim 0.16 \pm$ 0.02 arcmin $^{-2}$. The estimated number of members could also be increased by $\sim 18 \%$ to account for the incompleteness in our color selection, although we have not done so here. We also plot the cumulative number of $\mathrm{H} \alpha$-emitting $(\mathrm{EW}[\mathrm{H} \alpha]>40 \AA$ ) cluster members in $\mathrm{Cl} 0024+16$ from the survey by Kodama et al. (2004), which exhibits a similar rise to that exhibited by the $24 \mu \mathrm{m}$ population in this cluster. In contrast, MS 0451-03 shows little evidence for a strong mid-infrared population. The top panel illustrates the variation in survey area with radius in our two fields. This shows that the slower increase in the cumulative $24 \mu \mathrm{m}$ counts beyond $5 \mathrm{Mpc}$ in $\mathrm{Cl} 0024+16$ is purely due to the decline in coverage in the outskirts of our survey. The errors on our observations are the combination of counting statistics and the measured variance in the field number counts. The physical scale assumes an average redshift of $z=0.47$.

field counts as a function of survey area. This variance is included in the uncertainty in the field correction used in Figure 3.

By selecting $24 \mu \mathrm{m}$ sources with optical/near-infrared colors similar to those expected for cluster members, we have substantially reduced the field contamination in our sample. Figure 3 demonstrates a significant $24 \mu \mathrm{m}$ cluster population in $\mathrm{Cl} 0024+16$, with $155 \pm 18$ sources across our $25^{\prime} \times 25^{\prime}$ field (where the uncertainty includes the effects of clustering on the field correction). As can be seen, the excess population is distributed across the whole field, with the numbers continuing to increase to $\sim 5 \mathrm{Mpc}$, where the coverage of our data begins to decline. In contrast, in MS 0451-03 we find a much weaker excess, $28 \pm 17$, which is only marginally significant. Nevertheless, we note that our existing spectroscopic survey of this cluster (Fig. 2) confirms that there are $24 \mu \mathrm{m}$-detected cluster members, but the population appears much less numerous than in $\mathrm{Cl} 0024+16$. In both cases, the estimates of the total cluster populations could be increased by $\sim 18 \%$ to account for the incompleteness of our color selection, although to be conservative, we choose not to apply this correction.

While it is clear that there are populations of $24 \mu \mathrm{m}$ cluster members, we also need to consider lensing of background sources by these massive clusters, which may affect the predicted numbers of cluster members. Lensing has the effect of a boosting of flux and dilation of the projected area. To demonstrate this, we model the clusters as simple isothermal spheres. In this case, a background source will suffer an apparent deflection in radial direction by $\alpha \simeq\left(20^{\prime \prime}\right) \sigma_{1000}^{2}$, where $\sigma_{1000}$ is the cluster velocity disper- sion in units of $1000 \mathrm{~km} \mathrm{~s}^{-1}$. We adopt $\sigma=1150 \mathrm{~km} \mathrm{~s}^{-1}$ for $\mathrm{Cl} 0024+16$ and $\sigma=1290 \mathrm{~km} \mathrm{~s}^{-1}$ for MS 0451-03 (Trentham $\&$ Mobasher 1998). At a radius $r$, the source-plane area of an annular bin of width $d r$ will be $2 \pi(r-\alpha) d r$, and the flux of a lensed source will be boosted by a factor $r /(r-\alpha)$. We integrate the lensing prediction for our color-selected sample (taking $\sigma=$ $1200 \mathrm{~km} \mathrm{~s}^{-1}$, although the actual values may be lower, so this should be a conservative estimate) as a function of radius, finding that it could contribute $\lesssim 20$ sources to our samples; hence, in $\mathrm{Cl} 0024+16$ the excess signal we see is greater than can be explained by lensing. The situation with MS $0451-03$ is more problematic, although again we note that the spectroscopic identification of a handful of $24 \mu \mathrm{m}$ cluster members confirms that, as with $\mathrm{Cl} 0024+16$, there is a population of mid-infrared sources associated with the cluster.

\subsection{Star Formation}

To calculate the star formation rates (SFRs) for our mid-infrareddetected galaxies, we begin by estimating the total infrared luminosity over the rest-frame wavelength range $8-1000 \mu \mathrm{m}$. Since we only have MIPS $24 \mu \mathrm{m}$ photometry, we have to determine the correction factor required to convert our $24 \mu \mathrm{m}$ luminosities to total-infrared luminosities. To achieve this, we assume that the correlation between $15 \mu \mathrm{m}$ (corresponding to observed $\sim 24 \mu \mathrm{m}$ at $z \sim 0.5$ ) and total-infrared luminosity seen for local infrared galaxies (Chary \& Elbaz 2001) continues to hold at $z \sim 0.5$. We then follow a technique similar to that of Bell et al. (2005) and use the SED templates from Dale \& Helou (2002) to estimate the ratio of the observed-frame $24 \mu \mathrm{m}$ luminosity to the $8-1000 \mu \mathrm{m}$ luminosity. We calculate the ratio of total-infrared to observed $24 \mu \mathrm{m}$ luminosities for each SED template, taking the mean value of all the ratios as our correction factor and the range from the most active to the most quiescent SEDs as a conservative estimate of the systematic uncertainty. The correction factors are $16 \pm 2$ and $16 \pm 3$ for $\mathrm{Cl} 0024+16$ and MS 0451-03, respectively. We compare our conversion factors to the relation found by Chary \& Elbaz (2001) by calculating the mid-infrared to totalinfrared ratio, with the ISOCAM LW3 $(15 \mu \mathrm{m})$ filter for the Dale $\&$ Helou templates at $z=0.1$. The Chary \& Elbaz conversion for local luminous infrared galaxies (LIRGs) corresponds to $L_{\mathrm{IR}} \sim$ $\left(11_{-4}^{+6}\right) L_{15 \mu \mathrm{m}}^{0.998}$, while our estimate gives a prefactor of $7 \pm 3$ over the full range of LIRG templates. Thus, these two calibrations are consistent, although the reader should note that our estimates are roughly $40 \%$ fainter than would be given by Chary \& Elbaz (2001).

Our $5 \sigma, 24 \mu \mathrm{m}$ flux limit of $200 \mu \mathrm{Jy}$ corresponds to average total-infrared luminosities of $6 \times 10^{10}$ and $12 \times 10^{10} L_{\odot}$ in Cl 0024+16 and MS 0451-03, respectively; hence, the bulk of the populations we are detecting are LIRGs, with $L_{\mathrm{IR}} \geq 10^{11} L_{\odot}$. These luminosities translate into SFRs of $\sim 10$ and $\sim 20 M_{\odot} \mathrm{yr}^{-1}$, assuming the far-infrared star formation calibration given by Kennicutt (1998), with a Salpeter initial mass function (IMF) and a mass range of $0.1-100 M_{\odot}$.

In Figure 4 we plot the mid-infrared luminosity function for $\mathrm{Cl} 0024+16$, based on the color-selected sample out to a radius of $5 \mathrm{Mpc}$. To field-correct the luminosity function in $\mathrm{Cl} 0024+16$, we integrate the extrapolated power-law fit to the color-selected $24 \mu \mathrm{m}$ sources in the SWIRE ELAIS N1 field, from $\S 3.2$. We use the scatter in the normalization of $d N / d S$ in independent $25^{\prime} \times 25^{\prime}$ regions within the SWIRE field, combined with the counting errors in each bin, to give the uncertainty in the field correction. This plot demonstrates that the field-corrected luminosity function in $\mathrm{Cl} 0024+16$ is similar in form to that seen 


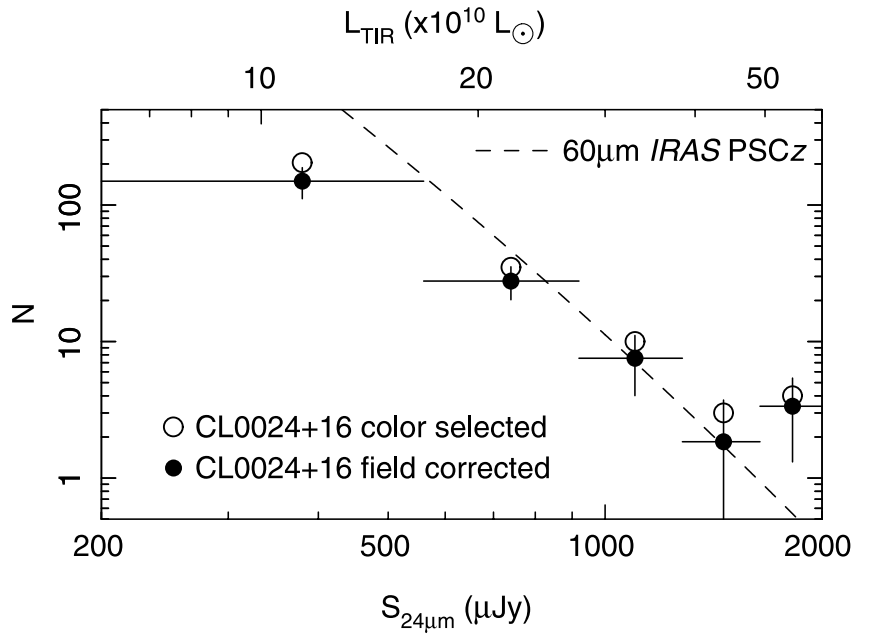

FIG. 4.- Infrared luminosity function for $\mathrm{Cl} 0024+16$, based on the photometrically selected cluster members and plotted with and without correction for residual field contamination. The total-infrared luminosities are derived by extrapolating the monochromatic MIPS $24 \mu \mathrm{m}$ flux, assuming the local $15 \mu \mathrm{m}-$ far-infrared correlation holds at $z \sim 0.5$ (see $\S 3.3$ ). The estimated number of $24 \mu \mathrm{m}$ cluster members in MS 0451-03 is insufficient to provide a similar plot for that cluster. For comparison, we plot the luminosity function of the $60 \mu \mathrm{m}$ IRAS Point Source Catalog Redshift survey from Takeuchi et al. (2003), with an arbitrary normalization. The cluster luminosity function is similar in shape to that of three local field galaxies, given the large uncertainties.

for $60 \mu \mathrm{m}$-selected populations at low redshift (Takeuchi et al. 2003).

While there are too few sources in MS 0451-03 to allow us to make an equivalent plot in Figure 4 for that cluster, we can use the $\mathrm{Cl} 0024+16$ luminosity function to estimate how many sources we would detect, if we placed the $\mathrm{Cl} 0024+16$ population at $z=0.55$. Applying a factor to scale for the effective areas of our surveys in $\mathrm{Cl} 0024+16$ and MS 0451-03, we estimate that we would detect $69 \pm 21$ galaxies above the luminosity limit, $12 \times 10^{10} L_{\odot}$, of the MS 0451-03 survey. This compares to our estimate of $28 \pm 17$ from our MS 0451-03 catalog, indicating roughly a factor of 2-3 difference in the numbers of mid-infrared sources in the two clusters. This suggests that, although the dearth of mid-infrared sources in MS 0451-03 may be partly due to sensitivity limitations, there may be a real difference in the mid-infrared populations in the two clusters.

\subsubsection{Mid-Infrared versus $\mathrm{H} \alpha$ Tracers of Activity}

We can also compare the properties of our mid-infraredselected sample in $\mathrm{Cl} 0024+16$ with the $\mathrm{H} \alpha$ survey of this cluster by Kodama et al. (2004). Kodama et al. obtained a deep image of the cluster in a narrowband filter, centered on redshifted $\mathrm{H} \alpha$. Galaxies with excess emission in this narrowband filter were identified as cluster members with $\mathrm{H} \alpha$ emission. They estimate that their sensitivity limit corresponds to a SFR of $\sim 1.5 M_{\odot} \mathrm{yr}^{-1}$, significantly below the estimated SFR limit of our mid-infrared survey, $\sim 10 M_{\odot} \mathrm{yr}^{-1}$.

To start with, we compare in Figure 3 the cumulative radial profile of the mid-infrared sources with the $\mathrm{H} \alpha$-emitting galaxies from Kodama et al. (2004). As can be seen, the two tracers show very similar trends, with a steep rise from $1 \mathrm{Mpc}$ out to $\sim 5 \mathrm{Mpc}$, beyond which our coverage declines. The reader should also note that the lack of mid-infrared coverage for the core of the cluster will contribute to the steeper drop of the $24 \mu \mathrm{m}$ counts in the innermost bin in Figure 3. Surprisingly, Figure 3 also shows that there are similar numbers of $\mathrm{H} \alpha$ emitters and $24 \mu \mathrm{m}-$ detected members, even though there is a significant difference in the

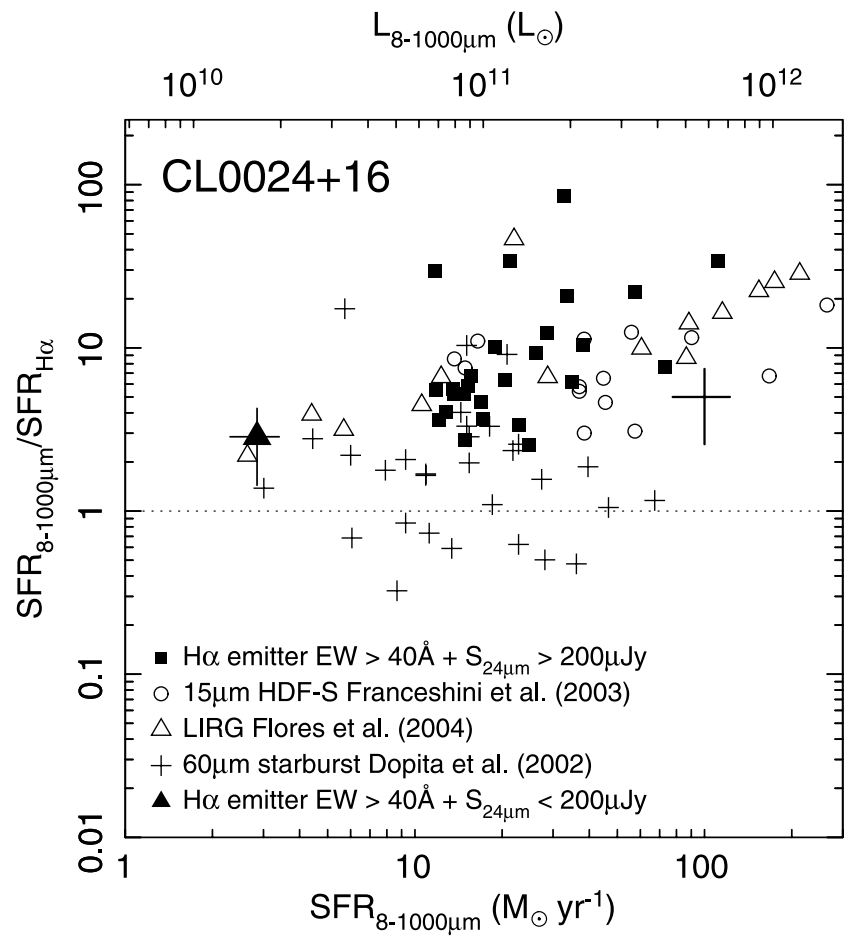

FIG. 5.-Comparison of the star formation rates derived from $\mathrm{H} \alpha$ (Kodama et al. 2004) and total-infrared luminosities for individual $\mathrm{H} \alpha$-detected cluster members in $\mathrm{Cl} 0024+16$. The ratio $\mathrm{SFR}(\mathrm{IR}) / \mathrm{SFR}(\mathrm{H} \alpha)$ provides an estimate of the level of obscuration of the star-forming regions within these galaxies. We see a typical offset of a factor of 5 between the infrared and $\mathrm{H} \alpha$ estimates (the latter have not been corrected for extinction), although there is a wide scatter. We also plot data from the literature of similar studies of infrared-luminous field galaxies from Flores et al. (2004), Franceshini et al. (2003), and Dopita et al. (2002), corrected to our cosmology. It appears that the level of obscuration of star-forming infrared cluster galaxies (in $\mathrm{Cl} 0024+16$ ) is similar to that of mid-infrared-selected field galaxies at this epoch. We do not plot individual error bars for clarity but show a representative error bar. Finally, to probe obscuration from faint mid-infrared sources, we stack the $24 \mu \mathrm{m}$ data for $\mathrm{H} \alpha$ emitters that are not detected in the MIPS image, yielding a $5 \sigma$ statistical detection. This confirms the general trend seen above our nominal luminosity limit that there appears to be an increase in the level of obscuration [SFR(IR)/SFR(H $\alpha)$ ] with infrared luminosity. [See the electronic edition of the Journal for a color version of this figure.]

relative sensitivity of the two surveys. Therefore, we next compare the estimates of the SFRs for individual galaxies from $\mathrm{H} \alpha$ and the mid-infrared, to relate these two star formation indicators for confirmed cluster members.

In Figure 5 we compare the star formation rates for $\mathrm{H} \alpha$ narrowband-selected sources and their $24 \mu \mathrm{m}$ counterparts. ${ }^{10}$ From this figure, it can be seen that the SFRs for the midinfrared-detected members, derived from the $\mathrm{H} \alpha$ emission line (but not corrected for extinction), appear to underestimate the SFRs from the mid-infrared by factors of $\gtrsim 5$. Including the standard assumption of 1 mag of extinction and correcting for a $30 \%$ contribution to the measured fluxes from [N II] (see Kodama et al. 2004) would reduce this offset to a factor of 2.5. However, such a simplistic correction would miss the fact that the discrepancy in SFRs appears to increase with infrared luminosity, such that the most active systems are also the most obscured.

To test for biases due to our $24 \mu \mathrm{m}$ sensitivity limit, we stack thumbnail images from the MIPS image of $\mathrm{Cl} 0024+16$ for $\mathrm{H} \alpha$ emitters from Kodama et al. (2004) that are not individually detected, i.e., $S_{24 \mu \mathrm{m}}<200 \mu \mathrm{Jy}$. We find a $\sim 5 \sigma$ detection with

10 The optical star formation rates are derived from the $\mathrm{H} \alpha$ and adjacent [ $\mathrm{N}$ II] $\lambda \lambda 6548,6583$ emission lines after Kennicutt et al. (1994). 
$S_{24 \mu \mathrm{m}}=57 \pm 11 \mu \mathrm{Jy}$. In terms of star formation, this corresponds to $\sim 3 M_{\odot} \mathrm{yr}^{-1}$. We plot this point on Figure 5, with the corresponding average $\mathrm{H} \alpha$-derived SFR for the stack, $\sim 1 M_{\odot} \mathrm{yr}^{-1}$. This point seems to be roughly consistent with the trend seen, that obscuration [i.e., $\operatorname{SFR}(\mathrm{IR}) / \mathrm{SFR}(\mathrm{H} \alpha)$ ] increases with the level of infrared activity.

We estimate that the correction required to bring the $\mathrm{H} \alpha$ derived SFRs in line with the infrared SFRs corresponds to a reddening of $A_{V} \sim 2.5$, slightly larger than the value used to explain the broadband colors of these galaxies on the $(R-K)-(B-R)$ color plane. This reddening estimate will also be affected by the choice of adopted SED when deriving total-infrared luminosities, although in this work, we have chosen a conversion that represents infrared galaxies of a wide range of activities, so our estimates are conservative. We also note that there is a factor of 40 range in infrared- to $\mathrm{H} \alpha$-derived SFRs within our sample, which is larger than expected from our measurement errors and indicates that there may be a wide range of extinction among the individual LIRGs; in part, probably due to the patchiness of the dust within these galaxies.

We also compare our relation between mid-infrared- and $\mathrm{H} \alpha$ derived SFRs to that seen in samples of field galaxies from the literature; a sample of LIRGs in the range $0.2<z<0.7$ from Flores et al. (2004), $15 \mu \mathrm{m}$-detected ISOCAM sources from the Hubble Deep Field-South at $0.2<z<1.5$ (Franceshini et al. 2003), and IRAS $60 \mu \mathrm{m}$ warm infrared galaxies from Dopita et al. (2002). The typical level of SFR(IR)/SFR( $\mathrm{H} \alpha)$ is similar to $\mathrm{H} \alpha /$ mid-IR-detected objects in $\mathrm{Cl} 0024+16$, suggesting that these cluster galaxies are similar in nature (at least in terms of their mix of star formation and obscuration) to star-forming LIRGs in the field, at similar epochs.

\subsection{Total Cluster Star Formation Rates}

We now estimate a total star formation rate for the clusters, using the typical SFRs of the likely star-forming mid-infrared members. Since we lack observations in the central $\sim 1 \mathrm{Mpc}$ in $\mathrm{Cl} 0024+16$ due to the unobserved GTO region, we also need to correct for this missing population, using the $15 \mu \mathrm{m} I S O$ observations from Coia et al. (2005).

\subsection{1. $\mathrm{Cl} 0024+16$}

As shown by the radial number counts presented in Figure 3, the $24 \mu \mathrm{m}$ members are widely distributed across the clusters out to a projected radius of $\sim 2 \mathrm{Mpc}$ (corresponding to $\sim R_{200}$ [Carlberg et al. 1997]; note that throughout, we use projected distances) and beyond. To compare the mid-infrared estimates of the total SFRs within representative regions of different clusters, we therefore adopt a $2 \mathrm{Mpc}$ radius for our calculations, which roughly matches the size of the surveyed regions in some of the earlier small-field surveys.

We integrate over the luminosity function in Figure 4 for sources with projected radii $<2 \mathrm{Mpc}$, correcting for the expected contamination from field galaxies, using the SWIRE data for the ELAIS N1 field (see $\S 3.2$ ). To account for the missing sources in the unobserved GTO core region, we need to add the SFRs in the $15 \mu \mathrm{m}$ members in this region found by Coia et al. (2005), after converting their $15 \mu \mathrm{m}$ fluxes to star formation rates using an identical method to that presented in $\S 3.3$.

Our derived total-infrared luminosities are lower than those derived by Coia et al. (2005) by a factor of $\sim 2$. This difference can be attributed to the different methods of conversion used. The method of Coia et al. involves extrapolating their observed $15 \mu \mathrm{m}$ photometry to $15 \mu \mathrm{m}$ in the rest frame, using an SED fitted to their optical and mid-infrared photometry. This is then converted to a total-infrared luminosity, using the empirical conversion of Chary \& Elbaz (2001) described above. In contrast, our observations correspond to $\sim 15 \mu \mathrm{m}$ in the rest frame. Hence, some of this offset is due to the differences in conversion from $15 \mu \mathrm{m}$ luminosity to total-infrared emission discussed in $\S 3.3$, and the remainder is due to the choice of SED by Coia et al. (2005), which overpredicted the observed $24 \mu \mathrm{m}$ fluxes. As we have stated earlier, our calculations should yield conservative limits on the SFRs. We note that, with our total-infrared calibration, the $5 \sigma$ detection limit of the Coia et al. (2005) ISO observations corresponds to $\sim 3 \times 10^{10} L_{\odot}$.

With our conversion, we find three galaxies in the Coia et al. (2005) sample that exceed our luminosity limit of $6 \times 10^{10} L_{\odot}$, so we can add their SFRs to our total, within $2 \mathrm{Mpc}$ of the core. We thus find a total star formation rate for the 40 sources within $2 \mathrm{Mpc}$ to be $1000 \pm 210 M_{\odot} \mathrm{yr}^{-1}$. The error is derived by bootstrap-resampling the flux distribution of sources within $2 \mathrm{Mpc}$ and integrating the new luminosity function. The median SFR per galaxy in our sample within $2 \mathrm{Mpc}$ is $\sim 16 M_{\odot} \mathrm{yr}^{-1}$. We note that correcting for the estimated incompleteness in our color selection of cluster members would increase our estimate of the total SFR by a factor of 1.07 , which corresponds to a change less than its quoted uncertainty.

For comparison, we also note that the $\mathrm{H} \alpha$ observations of Kodama et al. (2004) detect 100 galaxies within a similar region to that discussed here. They find a cumulative SFR of $\sim 470 M_{\odot} \mathrm{yr}^{-1}$, or about $4.7 M_{\odot} \mathrm{yr}^{-1}$ galaxy $^{-1}$, greater than their approximate sensitivity limit of $1.5 M_{\odot} \mathrm{yr}^{-1}$. Restricting the $\mathrm{H} \alpha$ sample to the $>10 M_{\odot} \mathrm{yr}^{-1}$ limit of our mid-infrared survey, we find that the $\mathrm{H} \alpha$ survey would yield an integrated SFR of $\sim 220 M_{\odot} \mathrm{yr}^{-1}$. This confirms the effect seen in Figure 4 that, although $\mathrm{H} \alpha$ and mid-infrared surveys are detecting a similar population, the $\mathrm{H} \alpha$ tracer severely underestimates the underlying activity in the most active sources in the cluster; these starbursting systems are producing dust at a more copious rate and therefore are optically obscured. In addition, we can state that the mid-infrared survey detects the bulk of the total star formation activity within this region, requiring only a $\sim 20 \%$ correction for the star formation activity in sources with $1-10 M_{\odot} \mathrm{yr}^{-1}$.

\subsection{2. $M S 0451-03$}

We have seen that, compared to $\mathrm{Cl} 0024+16$, this cluster appears to be deficient in mid-infrared sources (at least down to our luminosity limit). Nevertheless, even a small excess of midinfrared sources in the cluster could contribute a nonnegligible star formation rate to the overall activity of the cluster. We therefore estimate the total star formation rate within $\sim 2 \mathrm{Mpc}$, using the small number of excess sources identified by using our $(R-K)-(B-R)$ color-color selection. Applying the same approach as used for $\mathrm{Cl} 0024+16$, we find a total star formation rate within a $2 \mathrm{Mpc}$ radius of the cluster center of $200 \pm 100 M_{\odot} \mathrm{yr}^{-1}$. The median SFR within $2 \mathrm{Mpc}$ is $\sim 35 M_{\odot} \mathrm{yr}^{-1}$ galaxy $^{-1}$ (reflecting the brighter luminosity limit in this cluster).

In $\S 3.3$ we estimated the number of mid-infrared sources that we would detect if MS 0451-03 had the same mid-infrared luminosity function as $\mathrm{Cl} 0024+16$, taking into account the slightly different areal coverages between the clusters. Similarly, we can estimate the total star formation rate in MS 0451-03, if we reach a luminosity limit identical to $\mathrm{Cl} 0024+16$. Assuming that the faint end of the luminosity function in MS 0451-03 follows a shape similar to $\mathrm{Cl} 0024+16$, we estimate that the total star formation rate down to $6 \times 10^{10} L_{\odot}$ in this cluster is $<460 M_{\odot} \mathrm{yr}^{-1}$. Although the small number of excess sources in MS 0451-03 can lead to a relatively high star formation rate (compared to what 
might be found using an optical tracer, for example), there is a clear difference in the population of mid-infrared sources in the two clusters; but what is the physical cause of this?

Although the clusters are of similar mass and at a similar redshift, they differ strongly in their intracluster environments and dynamical status (Treu et al. 2003). Cl 0024+16 is dynamically active (Czoske et al. 2001; Kodama et al. 2004), and this may provide the impetus for the triggering of star formation via mergers and interactions of gas-rich spiral galaxies within the cluster, even in the apparently high-density core. The intracluster medium (ICM) of MS 0451-03 is much hotter and denser (by nearly an order of magnitude) than $\mathrm{Cl} 0024+16$, and this will have an impact on the radii within which processes such as starvation and ram pressure stripping operate (Treu et al. 2003). In MS 0451-03, the hot ICM will be much more effective at starving galaxies of their gas reservoirs at a larger clustocentric radii than in $\mathrm{Cl} 0024+16$. The relative dearth of mid-infrared sources in MS 0451-03 might then suggest that the active regions within the mid-infrared population are comparatively sensitive to these gas-removal mechanisms. Unlike $\mathrm{Cl} 0024+16$, unfortunately there are no published optical SFR studies of MS 0451-03 to confirm a wholesale decline in the SFR in this cluster. Nevertheless, the small excess of mid-infrared sources in MS 0451-03 shows that it hosts quite significant star formation, so it is unclear at this stage exactly what physical processes control the distribution of star-forming galaxies in different types of clusters. We will address the issue in our next paper, in which we will study the properties of the $24 \mu \mathrm{m}$ cluster members in more detail, using the available spectroscopy, dynamics, and HST imaging (J. E. Geach et al. 2006, in preparation; see also S. M. Moran et al. 2006, in preparation).

\section{OBSCURED STAR FORMATION IN CLUSTERS OUT TO $z \sim 1$}

Virialized regions of the universe are currently dominated by passive galaxies: elliptical and lenticular galaxies, with the main contribution to the global star formation rate density at $z=0$ coming from late-type spiral galaxies in low-density environments. However, at higher redshifts, the high-density environments within clusters show increasing numbers of actively star-forming galaxies, possibly reflecting an increase in the frequency of activity similar to that seen in the surrounding field. Moreover, it is clear that there is significant hidden star formation in clusters in this redshift range. Observations by ISO and now our new Spitzer observations reveal populations of infrared galaxies in clusters at low and intermediate redshift, with SFRs much higher than would be measured using optical tracers such as $\mathrm{H} \alpha$ or [O II] 23727, due to the extinction effect of dust.

Given this new information, what is the evolution of the total star formation rates in massive clusters; i.e., how does the increase in activity in high-density environments at high redshift behave in the mid-infrared? To compare the total mid-infrared-derived star formation rate in clusters from $z=0-1$, we use the total SFR within $\sim 2 \mathrm{Mpc}$ and normalize by the best-estimate mass of the cluster (for example, see Kodama et al. 2004). For Cl 0024+16 and MS 0451-03, these are $6.1 \times 10^{14} M_{\odot}($ Kneib et al. 2003) and $15 \times 10^{14} M_{\odot}$ (La Roque et al. 2003), respectively, where the masses are within $2 \mathrm{Mpc}$ of the clusters' centers.

To build up a history of star formation in clusters out to $z \sim 1$, we compare our results to previous ISO and IRAS studies of the clusters: Perseus (A426), A1689, A370, A2218, and J1888.8 CL (Cl 0054-27), summing the known cluster member's SFRs down to a far-infrared limit of $6 \times 10^{10} L_{\odot}$, the limit of the $\mathrm{Cl}$ $0024+16$ observations. To ensure correct comparison of the rates, if necessary we rederive the SFRs, using our employed calibration from Kennicutt (1998), with a standard Salpeter IMF with a mass range $0.1-100 M_{\odot}$. For the $I S O$ observations, we also rederive the far-infrared $8-1000 \mu \mathrm{m}$ luminosity with the method we present above, using the bandpass of the ISO LW3 filter to convert mid- to total-infrared. The ISO observations of these clusters are summarized in the review by Metcalfe et al. (2005). Note that the previous ISO and IRAS observations have concentrated on the core regions of clusters (typically within $1 \mathrm{Mpc}$ ), so we restrict our star formation integration to within a radius of $2 \mathrm{Mpc}$ in $\mathrm{Cl} 0024+16$ and MS 0451-03 and compare to estimates of the total star formation within the equivalent radius in the other clusters. In the case of MS 0451-03, we estimate an upper limit to the star formation rate extrapolated to a luminosity limit of $6 \times 10^{10} L_{\odot}$ by using the method described in $\S 3.4 .2$, assuming that the faint end of the luminosity function follows a similar shape to that in $\mathrm{Cl} 0024+16$. We estimate that the upper limit to the total star formation rate down to $6 \times 10^{10} L_{\odot}$ in MS $0451-03$ is $\lesssim 460 M_{\odot} \mathrm{yr}^{-1}$.

At low redshifts, we use the nearby rich cluster Perseus, observed with IRAS (Meusinger et al. 2000). We sum over the known cluster members with $L_{\mathrm{FIR}}>6 \times 10^{10} L_{\odot}$ and convert their luminosities to SFRs, giving a lower limit to the total star formation rate in the cluster of $>22 M_{\odot} \mathrm{yr}^{-1}$ over an area equivalent to $10 \mathrm{deg}^{2}$, which in terms of physical coverage is similar to the $2 \mathrm{Mpc}$ radius used in $\mathrm{Cl} 0024+16$ and MS $0451-03$. We use the mass estimate of Ettori et al (2002) of $3.1 \times 10^{14} M_{\odot}$ and extrapolate to $2 \mathrm{Mpc}$ to determine the normalized star formation rate per mass to be $\sim 3 M_{\odot} \mathrm{yr}^{-1} / 10^{14} M_{\odot}$.

Fadda et al. (2000) and Duc et al. (2002) observed A1689 $(z=0.18)$ at $15 \mu \mathrm{m}$, with $I S O$ detecting 16 cluster members within $0.5 \mathrm{Mpc}$ of the core. With the mid-infrared to total-infrared conversion used in this work, we find two galaxies (detected with the LW3 $15 \mu \mathrm{m}$ filter) above our luminosity limit. In order to estimate the star formation expected out to a radius of $2 \mathrm{Mpc}$, we assume a radial profile for the mid-infrared population similar to that found for $\mathrm{Cl} 0024+16$ and extrapolate the star formation rate from within a radius of $0.5-2 \mathrm{Mpc}$. The resulting star formation rate is $\sim 280 M_{\odot} \mathrm{yr}^{-1}$ within $2 \mathrm{Mpc}$ of the core. We use the mass from King et al. (2002), correcting to $2 \mathrm{Mpc}$, yielding a mass-normalized value of $\sim 30 M_{\odot} \mathrm{yr}^{-1} / 10^{14} M_{\odot}$. A2218 is another rich cluster at a similar redshift to A1689, with $z=0.175$; however, the mid-infrared activity in this structure is much lower than in A1689. The $15 \mu \mathrm{m}$ observations of Biviano et al. (2004) found nine members within a similar radius ( $\lesssim 0.4 \mathrm{Mpc}$ ), but only one of these corresponds to a star-forming galaxy, and the median infrared luminosity is only $6 \times 10^{8} L_{\odot}$, or just $0.1 M_{\odot} \mathrm{yr}^{-1}$. A370 is at a similar redshift to $\mathrm{Cl} 0024+16$ at $z=0.37$ but also appears deficient in LIRGs. Only one cluster member was detected at $15 \mu \mathrm{m}$. We plot these points as upper limits using our luminosity limit, again extrapolating to account for radial coverage out to $2 \mathrm{Mpc}$ using the shape of the radial profile of mid-infrared sources in $\mathrm{Cl} 0024+16$. We use the cluster masses from Pratt et al. (2005) and Girardi \& Mezzetti (2001) for A2218 and A370, respectively, extrapolating to find the mass within $2 \mathrm{Mpc}$. The mass-normalized upper limit for $\mathrm{A} 2218$ is $30 M_{\odot} \mathrm{yr}^{-1} / 10^{14} M_{\odot}$ and for $\mathrm{A} 370$, $9 M_{\odot} \mathrm{yr}^{-1} / 10^{14} M_{\odot}$.

$\mathrm{J} 1888.16(\mathrm{Cl} 0054-27)$ is at $z=0.56$ and was observed by Duc et al. (2004) using ISOCAM at $15 \mu \mathrm{m}$. Six mid-infrared sources were detected that are confirmed cluster members, while a further two have redshifts suggesting they are members of infalling groups at a slightly higher redshift. Using the calibration of Chary \& Elbaz (2001), Duc et al. find that all of their detected members are LIRGs, with inferred $L_{\text {FIR }}>1.3 \times 10^{11} L_{\odot}$, and with individual 


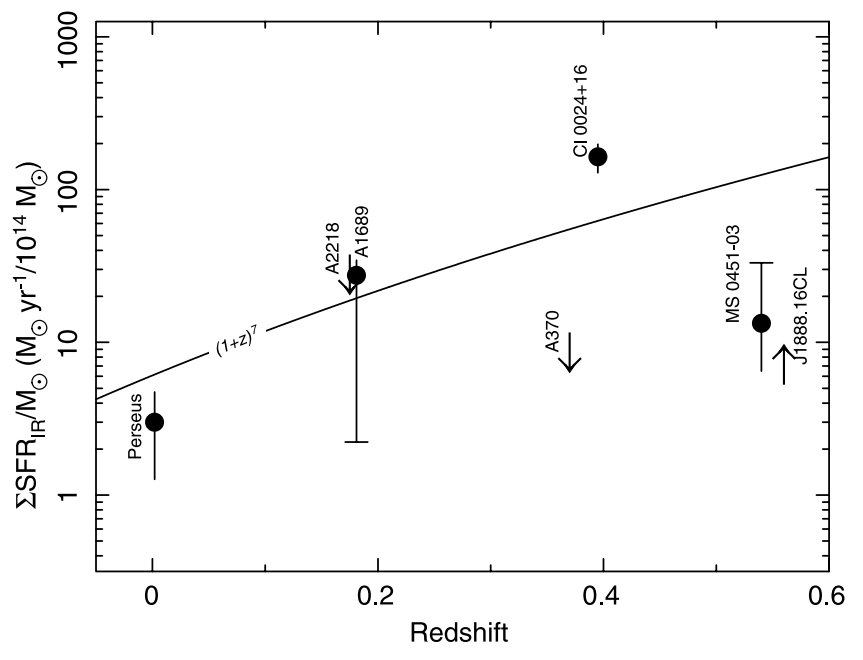

FIG. 6.-Variation in the mass-normalized star formation rates in clusters out to $z \sim 0.5$. The SFRs are from the mid-infrared populations within $\sim 2 \mathrm{Mpc}$, and these are normalized to the best estimate of the cluster mass, which is derived via lensing estimates or from the X-ray luminosities. We also plot an evolutionary model for the counts of star-forming ULIRGs from Cowie et al. (2004), normalized to the mean star formation rate in $\mathrm{Cl} 0024+16$ and MS 0451-03 (again we note that these could be increased by $\sim 18 \%$ to account for incompleteness in our color selection of cluster members). The MS 0451-03 point has an upper limit that corresponds to the extrapolated estimate for the total star formation down to the luminosity limit in $\mathrm{Cl} 0024+16\left(6 \times 10^{10} L_{\odot}\right)$, while the data point shows the cluster's summed SFR down to the limit of our data $\left(12 \times 10^{10} L_{\odot}\right)$. There is evidence for an increasing rate of activity in more distant clusters, as traced through their midinfrared populations. However, there is also clear evidence for a wide variation in activity in even massive clusters at a similar epoch. This suggests that the mid-infrared populations are sensitive tracers of environmental changes within the clusters. The errors on the total star formation rates for $\mathrm{Cl} 0024+16$ and MS 0451-03 are derived from bootstrap resampling of the mid-infrared distribution, whereas for Perseus and A1689, the errors are from counting statistics. Note that there are systematic uncertainties in all of the estimates, depending on the specific choice of SED in the conversion from mid-infrared luminosity to SFR (the plotted uncertainties also do not take into account the errors in the cluster mass estimates). Upper limits are equivalent star formation rates for one detected at our luminosity limit of $6 \times 10^{10} L_{\odot}$ (and extrapolated to account for the coverage difference in the survey) in those clusters where no sources were detected above this limit.

SFRs in the range $20-120 M_{\odot} \mathrm{yr}^{-1}$. We recalibrate the SFR using the method set out in $\S 3.3$ and find a conservative lower limit to the total cluster star formation rate of $>70 M_{\odot} \mathrm{yr}^{-1}$. The mass of the cluster is determined by Girardi \& Mezzetti (2001), and we extrapolate to $2 \mathrm{Mpc}$, giving a total SFR $>7 M_{\odot} \mathrm{yr}^{-1} / 10^{14} M_{\odot}$.

We present the results in Figure 6, in which we plot the sum of the SFRs within $\sim 2$ Mpc of the clusters' cores, normalized using the estimated total mass of the cluster (based on either lensing or X-ray models). There is strong evolution in the star formation rates in the clusters out to high redshifts, but it is important to note that several clusters seem to have very low levels of activity, below our luminosity limits. For example, Cl 0024+16 appears significantly more active than A370, which is at an almost identical redshift. This might point to differing environmental influences between clusters as being the dominant influence on the star formation histories of infalling galaxies. Nevertheless, assuming that we have only selected those clusters with significant activity in Figure 6, there still appears to be strong evolution in the sample out to $z \sim 0.5$. This observation could simply reflect the increase in the number of star-forming galaxies seen in clusters and the field out to this redshift (with these obscured systems representing the high-luminosity tail of the general "blue" population). The idea that the cluster (obscured) star formation histories mimic that of the field is supported by the rough consistency with the $(1+z)^{7}$ trend found by Cowie et al. (2004) for the number of star-forming ultraluminous infrared galaxy (ULIRG) radio sources out to $z \sim 1.5$. Kodama et al. (2004) present a similar analysis for the evolution of the $\mathrm{H} \alpha$-derived star formation in clusters out to $z \sim 1$. They find relatively strong evolution in the total SFRs in clusters over this range, $\sim(1+z)^{4}$, but as in this study, there is considerable scatter in the total star formation rates between clusters, even after mass normalization. This hints that, although there might be a nominal rise in the level of star formation in clusters out to $z \sim 1$, this is mitigated by the fact that individual cluster environments have a strong influence on the star formation histories of their constituent galaxies. This scatter is due to the complexity of processes operating solely in the dense environments. A detailed study of the sources in $\mathrm{Cl} 0024+16$ and MS 0451-03 may elucidate this issue.

\section{CONCLUSIONS AND SUMMARY}

We have used the MIPS instrument on Spitzer to survey the $24 \mu \mathrm{m}$ populations of two optically rich clusters at $z \sim 0.5$ : $\mathrm{Cl} 0024+16$ and MS 0451-03. The samples are $\sim 80 \%$ complete at $200 \mu \mathrm{Jy}$, corresponding to total-infrared $(8-1000 \mu \mathrm{m})$ luminosities of $6 \times 10^{10}$ and $12 \times 10^{10} L_{\odot}$ at $z=0.39$ and 0.55 , respectively, equivalent to minimum SFRs of $\sim 10$ and $\sim 20 M_{\odot} \mathrm{yr}^{-1}$. We detect a total of 986 and 1018 mid-infrared sources above this flux limit across $\sim 25^{\prime} \times 25^{\prime}$ fields in $\mathrm{Cl} 0024+16$ and MS 0451-03. Our observations probe from around the cores out to the turnaround radius at $\sim 5 \mathrm{Mpc}$, where the clusters merge into the field. In MS 0451-03, we also analyze archival MIPS observations of the central $\sim 5^{\prime} \times 5^{\prime}$ of the cluster, which our observations had to avoid. Similarly, in $\mathrm{Cl} 0024+16$, we make use of an ISO $15 \mu \mathrm{m}$ survey from Coia et al. (2005) in the central region to build up a picture of the distribution of mid-infrared sources over the complete range of cluster environments.

We exploit optical/near-infrared colors for the mid-infrared sources to reduce the background field contamination. We find a statistical excess of mid-infrared sources (within $\sim 5 \mathrm{Mpc}$ of the cluster core) at $S_{24 \mu \mathrm{m}}>200 \mu \mathrm{Jy}$ associated with $\mathrm{Cl} 0024+16$, $155 \pm 18$. In contrast, MS 0451-03 has a less significant population of mid-infrared sources, $28 \pm 17$, although we note that there are a small number of confirmed $24 \mu \mathrm{m}$ members in MS 0451-03 in our ongoing spectroscopic survey of this cluster.

Using our deep optical and near-infrared imaging of both clusters, we show that the $24 \mu \mathrm{m}$ sources in $\mathrm{Cl} 0024+16$ are mostly associated with star-forming galaxies with typically blue $(B-R)$ colors but can be dust-reddened by up to $A_{V} \sim 2.5 \mathrm{mag}$. We also compare the infrared star formation rate to that derived from an optical narrowband $\mathrm{H} \alpha$ survey of this cluster from Kodama et al. (2004). Typically, the $\mathrm{H} \alpha$-derived rates underestimate the extinction-free infrared rate by a factor of $\gtrsim 5$, suggesting significant obscuration of the activity in this cluster. We find that the level of obscuration for these individual cluster galaxies is comparable to that found for LIRGs in the field at similar epochs. This suggests that starbursts in clusters are similar (at least in terms of extinction) to those triggered in low-density environments. However, the variation in the $24 \mu \mathrm{m}$ populations of $\mathrm{Cl} 0024+16$ and MS 0451-03 suggests that the range of triggering and suppression mechanisms in clusters is complex.

We estimate that the total star formation rate (derived from the infrared) in the central region of $\mathrm{Cl} 0024+16\left(\lesssim R_{200}\right)$ is $1000 \pm 210 M_{\odot} \mathrm{yr}^{-1}$. MS $0451-03$ is much poorer in midinfrared sources, and we derive a total star formation rate estimate by summing over the small excess of objects, giving a total star formation rate of $200 \pm 100 M_{\odot} \mathrm{yr}^{-1}$ within the same physical radius. We note, however, that our mid-infrared survey can miss 
some star formation, if a substantial number of lower luminosity galaxies also exist in these clusters (as shown by the $\mathrm{H} \alpha$ survey of Kodama et al. 2004). However, we show that the majority of the activity is dominated by these dusty starbursts.

Finally, we look at the evolution of the specific SFR per cluster, with redshift $z \sim 0-0.5$, using our new estimates for the total star formation rates in $\mathrm{Cl} 0024+16$ and MS 0451-03. We compare these to estimates for lower and comparable redshift clusters studied with $I S O$ and IRAS. We find that the high-redshift clusters tend to have larger total star formation rates compared to the more quiescent low-redshift ones, with an evolution similar to that of field LIRGs. However, there is considerable scatter in this relation, and the evolution may only apply to the most active clusters. Although it is still unclear exactly what processes govern the star formation histories of rich clusters, this study has shown that rich environments can sustain significant amounts of hidden star formation and that this seems to increase at least out to $z \sim 0.5$. This hidden activity may have a profound influence on the life cycle of galaxies in high-density regions and the formation of the passive galaxy populations, elliptical and S0 galaxies that currently inhabit these environments. We will investigate these issues in more detail in our next paper, where we bring together spectroscopic and morphological information on the $24 \mu \mathrm{m}$ population in these fields. We will also extend our survey with new panoramic observations of distant clusters with Spitzer Cycle 3 GO time, using both MIPS and the IRS.

We thank the anonymous referee, whose comments greatly improved the clarity of this paper. This study is based on observations made with the Spitzer Space Telescope, which is operated by the Jet Propulsion Laboratory, California Institute of Technology, under a contract with NASA. This work also made use of the Spitzer Archive, which is operated by the Spitzer Science Center. J. E. G. is supported by a UK Particle Physics and Astronomy Research Council studentship; I. R. S. and G. P. S. acknowledge support from the Royal Society.

\section{APPENDIX}

\section{MATCHING OF MID-INFRARED AND OPTICAL CATALOGS}

Here we provide a brief description of the technique that we have adopted for identifying the optical counterparts to the midinfrared sources in our catalog. We have chosen to apply the technique of de Ruiter et al. (1977), who use a Bayesian estimator to identify the probability, $p(\mathrm{id} \mid r)$, of whether an optical source in close proximity to a mid-infrared source is likely to be a true match rather than a chance unrelated object.

First we parameterize the radial distances of sources in terms of a dimensionless variable,

$$
r=\left(\frac{\Delta \alpha^{2}}{\sigma_{\alpha}^{2}}+\frac{\Delta \delta^{2}}{\sigma_{\delta}^{2}}\right)^{1 / 2}
$$

where $\Delta \alpha$ and $\Delta \delta$ are the positional offsets of the sources in right ascension and declination (in the sense of mid-infrared-optical), and $\sigma_{\alpha}$ and $\sigma_{\delta}$ are the uncertainties in the positions (we assume that the optical uncertainties are negligible). A likelihood ratio LR can be constructed representing the probability of finding a genuine match (id) compared to the probability of finding a confusing (c) source within a radius $r$,

$$
\mathrm{LR}(r)=\frac{p(r \mid \mathrm{id})}{p(r)}=\frac{1}{2 \lambda} \exp \left[\frac{r^{2}}{2}(2 \lambda-1)\right],
$$

where $\lambda=\pi \sigma_{\alpha} \sigma_{\delta} \Sigma$, with $\Sigma$ representing the surface density of optical sources down to some limit. The physical interpretation of equation (A2) is that we are searching for a trade-off between finding a confusing background source, the distribution of which is governed by Poisson statistics, and the probability that the genuine match is located within $d r$ of the reference source; this is described by the Rayleigh distribution.

In our case $\lambda \sim 20 \Sigma$, and since we know that a cluster exists and the surface density of sources will not be uniform across the frame, we calculate the surface density of $R$-band sources brighter than the nearest neighbor, $\Sigma\left(m>m_{\mathrm{NN}}\right)$, in annular bins of width $1^{\prime}$ centered on the cluster at the radial distance of each mid-infrared source. The final part of the calculation requires us to calculate $p$ (id $\mid r$ ); having found a source at some radius $r$, what is the probability that it is the genuine match? Bayes' theorem provides a way to estimate this value, but first we require an a priori estimate, namely, what fraction $\theta$ of mid-infrared sources have optical counterparts? To estimate $\theta$ we count the number of sources with LR $>1$ over the frame, denoting these "matches," and compare to the number with LR $<1$. The value of $\theta$ is insensitive to the choice of likelihood threshold and is approximately 0.77 for $\mathrm{Cl} 0024+16$ and MS $0451-03$. We can then find

$$
p(\text { id } \mid r)=\frac{X \operatorname{LR}(r)}{X \operatorname{LR}(r)+1},
$$

where $X=\theta /(1-\theta)$. We define a positive match of sources at some cutoff value $L$ of the likelihood ratio, which we optimize to provide the best completeness $C$ and reliability $R$ of the sample:

$$
\begin{aligned}
C & =1-\sum_{\mathrm{LR}_{i}<L} p_{i}(\mathrm{id}) / N_{\mathrm{id}}, \\
R & =1-\sum_{\mathrm{LR}_{i}>L} p_{i}(c) / N_{\mathrm{id}}
\end{aligned}
$$


with $N_{\text {id }}=\sum p($ id). We choose a value of $p($ id $)=0.82$ for positive matches, corresponding to $R=C \sim 97 \%$ in both clusters. To improve the matching algorithm, we perform an identical calculation for the next-nearest neighbor to the mid-infrared sources. Thus, for each MIPS source we have a matching probability for both its nearest and next-to-nearest optical neighbors. This allows us to flag possible mergers (where both probabilities exceed our match threshold). If $p_{\mathrm{NNN}}>p_{\mathrm{NN}}$ then we choose the next-nearest neighbor as the genuine match.

\section{REFERENCES}

Balogh, M. L., Morris, S. L., Yee, H. K.. C., Carlberg, R. G., \& Ellingson, E. 1999, ApJ, 527, 54

Bell, E. F., Baugh, C. M., Cole, S., Frenk, C. S., \& Lacey, C. G. 2003, MNRAS, 343, 367

Bell, E. F., et al. 2005, ApJ, 625, 23

Bertin, E., \& Arnouts, S. 1996, A\&AS, 117, 393

Best, P. N. 2000, MNRAS, 317, 720

Biviano, A., et al. 2004, A\&A, 425, 33

Carlberg, R. G., Yee, H. K. C., \& Ellingson, E. 1997, ApJ, 478, 462

Chary, R., \& Elbaz, D. 2001, ApJ, 556, 562

Chary, R. et al. 2004, ApJS, 154, 80

Coia, D., et al. 2005, A\&A, 431, 433

Couch, W. J., Barger, A. J., Smail, I., Ellis, R. S., \& Sharples, R. M. 1998, ApJ, 497, 188

Cowie, L. L., Barger, A. J., Fomalont, E. B., \& Capak, P. 2004, ApJ, 603, L69

Czoske, O., Kneib, J.-P., Soucail, G., Bridges, T. J., Mellier, Y., \& Cuillandre, J.-C. 2001, A\&A, 372, 391

Dale, D. A., \& Helou, G. 2002, ApJ, 576, 159

de Ruiter, H. R., Arp, H. C., \& Willis, A. C. 1977, A\&AS, 28, 211

Dopita, M. A., Pereira, M., Kewley, L. J., \& Capaccioli, M. 2002, ApJS, 143, 47

Dressler, A. 1980, ApJS, 42, 565

Dressler, A., et al. 1997, ApJ, 490, 577

Duc, P.-A., Brinks, E., Springel, V., Pichardo, B., Weilbacher, P., \& Mirabel, I. F. 2000, AJ, 120, 1238

Duc, P.-A., et al. 2002, A\&A, 382, 60

2004, in IAU Colloq. 195, Outskirts of Galaxy Clusters: Intense Life in the Suburbs, ed. A. Diaferio (San Francisco: ASP), 347

Ettori, S., De Grande, S., \& Molendi, S. 2002, A\&A, 391, 841

Fadda, D., Elbaz, D., Duc, P.-A., Flores, H., Franceschini, A., Cesarsky, C. J., \& Moorwood, A. F. M. 2000, A\&A, 361, 827

Fasano, G., Poggianti, B. M., Couch, W. J., Bettoni, D., Kjærgaard, P., \& Moles, M. 2000, ApJ, 542, 673

Flores, H., Hammer, F., Elbaz, D., Cesarsky, C. J., Liang, Y. C., Fadda, D., \& Gruel, N. 2004, A\&A, 415, 885

Franceshini, A., et al. 2003, A\&A, 403, 501

Gerken, B., Ziegler, B., Balogh, M., Gilbank, D., Fritz, A., \& Jager, K. 2004, A\&A, 421, 59

Girardii, M., \& Mezzetti, M. 2001, ApJ, 548, 79

Hogg, D. W. 2001, AJ, 121, 1207

Kennicutt, R. C., Jr. 1998, ARA\&A, 36, 189

Kennicutt, R. C., Jr., Tamblyn, P., \& Congdon, C. E. 1994, ApJ, 435, 22
King, C. R., \& Ellis, R. S. 1985, ApJ, 288, 456

King, L. J., Clowe, D. I., \& Schneider, P. 2002, A\&A, 383, 118

Kneib, J.-P., et al. 2003, ApJ, 598, 804

Kodama, T., Balogh, M., Smail, I., Bower, R. G., \& Nakata, F. 2004, MNRAS, 354,1103

Kodama, T., \& Smail, I. 2001, MNRAS, 326, 637

Kodama, T., et al., 2005, PASJ, 57, 309

LaRoque, S. J., et al. 2003, ApJ, 583, 559

Lonsdale, C. J., et al, 2003, PASP, 115, 897 2004, ApJS, 154, 54

Makovoz, D., \& Khan, I., 2005, in ASP Conf. Ser. 347, Astronomical Data Analysis Software and Systems XIV, ed. P. L. Shopbell, M. C. Britton, \& R. Ebert (San Francisco: ASP), 81

Marleau, F. R., et al. 2004, ApJS, 154, 66

Metcalfe, L., Fadda, D., \& Biviano, A. 2005, Space Sci. Rev., 119, 425

Metcalfe, L., et al. 2003, A\&A, 407, 791

Meusinger, H., Brunzendorf, J., \& Krieg, R. 2000, A\&A, 363, 933

Moran, S. M., Ellis, R. S., Treu, T., Salim, S., Rich, R. M., Smith, G. P., \& Kneib, J.-P. 2006, ApJ, 641, L97

Moran, S. M., Ellis, R. S., Treu, T., Smail, I., Dressler, A., Coil, A. L., \& Smith, G. P. 2005, ApJ, 634, 977

Oemler, A. J. 1974, ApJ, 194, 1

Papovich, C., et al. 2004, ApJS, 154, 70

Poggianti, B. M., Bressan, A., \& Franceschini, A. 2001, ApJ, 550, 195

Poggianti, B. M., \& Wu, H. 2000, ApJ, 529, 157

Poggianti, B. M., et al. 1999, ApJ, 518, 576

Pratt, G. W., Bohringer, H., \& Finoguenov, A. 2005, A\&A, 433, 777

Smail, I., Dressler, A., Couch, W. J., Ellis, R. S., Oemler, A. J., Butcher, H., \& Sharples, R. M. 1997, ApJS, 110, 213

Smail, I., Morrison, G., Gray, M. E., Owen, F. N., Ivison, R. J., Kneib, J.-P., \& Ellis, R. S. 1999, ApJ, 525, 609

Smith, G. P., Treu, T., Ellis, R. S., Moran, S. M., \& Dressler, A. 2005, ApJ, 620 , 78

Takeuchi, T. T., Yoshikawa, K, \& Ishii, T. T. 2003, ApJ, 587, L89

Trentham, N., \& Mobasher, B. 1998, MNRAS, 299, 98

Treu, T., et al. 2003, ApJ, 591, 53

van Dokkum, P. G. 2005, AJ, 130, 2647

van Dokkum, P. G., \& Ellis, R. S. 2003, ApJ, 592, L53

Webb, T. M. A., Tee, H. K. C., Ivison, R. J., Hoekstra, H., Gladders, M. D.,

Barrientos, L. F., \& Hsieh, B. C. 2005, ApJ, 631, 187

Wilson, J. C., et al. 2003, Proc. SPIE, 4841, 451 